Arq. Bras. Med. Vet. Zootec., v.60, n.2, p.335-340, 2008

\title{
Neurectomia digital em eqüinos: comparação das técnicas guilhotina e stripping
}

\author{
[Digital neurectomy in horses: comparison of guillotine and stripping techniques] \\ R.R. Faleiros ${ }^{1}$, J.J.R. Tinto $^{2}$, A. Escobar ${ }^{3}$, G.E.S. Alves ${ }^{1}$ \\ ${ }^{1}$ Escola de Veterinária - UFMG \\ Caixa Postal 567 \\ 30123-970 - Belo Horizonte, MG \\ ${ }^{2}$ Curso de Medicina Veterinária - PUC-Minas - Betim, MG \\ ${ }^{3}$ Aluno de pós-graduação - FCAV-UNESP - Jaboticabal, SP
}

\begin{abstract}
RESUMO
Compararam-se as dificuldades transcirúrgicas e as complicações pós-operatórias das técnicas guilhotina (TG) e stripping (TS) para a neurectomia digital em eqüinos. Sob anestesia com halotano, quatro éguas tiveram os nervos digitais de um dos membros torácicos e um dos pélvicos submetidos à TG, enquanto os nervos digitais dos membros colaterais foram submetidos à TS. Os tempos cirúrgicos médios de TG e TS foram semelhantes. $\mathrm{O}$ comprimento médio do fragmento do nervo removido foi três vezes maior em TS $(\mathrm{P}<0,001)$. Independente da técnica utilizada, houve perda total da sensibilidade nos talões de todos os membros dentro dos quatro primeiros meses da cirurgia. Após 14 meses, houve retorno da sensibilidade em $37 \%$ dos membros em TG e $18,8 \%$ em TS $(\mathrm{P}=0,06)$. Ao exame de palpação para identificação de neuromas dolorosos, houve episódios de sensibilidade discreta em um maior número de cotos nervosos proximais operados com TS, 53,6\% contra $6,4 \%$ dos operados com TG $(\mathrm{P}=0,003)$. Ambas as técnicas foram satisfatórias por não apresentarem maiores complicações durante ou após a cirurgia. Considerou-se que TG apresentou menor potencial para produzir reinervação e neuromas dolorosos.
\end{abstract}

Palvaras-chave: eqüino, neurectomia, dor, nervo, reinervação

\begin{abstract}
The surgical difficulties and the postoperative complications of two techniques for equine digital neurectomy, the guillotine (TG) and the stripping (TS), were evaluated. Four mares under halothane anesthesia were submitted to the TS in the digital nerves of a thoracic and a pelvic limbs, and TG in the contralateral limbs. The mean surgical times for TG and TS were similar. The mean transected nerve length was three times greater in TS $(P<0.001)$. In both techniques, total lost of sensibility was observed in all heel bulbs during the first four months. After 14 months, sensibility returned to $37 \%$ limbs in treated by TG, and $18.8 \%$ in those treated by TS $(P=0.06)$. Discreet painful sensation was identified by the response of digital palpation of the neuroma in a greater number of nerve stumps where TS was performed, 53.6\% versus $6.4 \%$ of those operated by TG $(P=0.003)$. No major complications were observed during or after surgery and both techniques were considered satisfactory. TG was considered with less potential to produce painful neuromas and reinnervation.
\end{abstract}

Keywords: horse, neurectomy, pain, nerve, reinnervation

\section{INTRODUÇÃO}

A terapia da dor tem sido um dos grandes desafios da medicina, tanto na área humana como na veterinária. Apesar do grande avanço farmacológico observado nos últimos anos, que vem contribuindo sensivelmente para o tratamento da dor aguda, ainda não existem fármacos disponíveis, totalmente seguros e livres de efeitos colaterais, que possam ser usados quando a dor adquire caráter crônico. Além disso, a cronicidade álgica ultrapassa os limites

Recebido em 27 de julho de 2007

Aceito em 10 de janeiro de 2008

E-mail: faleiros@ufmg.br 
autoprotetores da dor e promove uma série de modificações fisiológicas e psicológicas no indivíduo, colocando-o em uma situação de sofrimento constante e tornando os sintomas e efeitos dolorosos extremamente complexos e de difícil acesso terapêutico (Faleiros et al., 1997). Assim, em casos severos, o tratamento mais efetivo ainda é a interrupção temporária da transmissão nervosa do local afetado por aplicação de anestésicos locais ou neurolíticos, ou a interrupção definitiva por secção ou remoção cirúrgica do nervo, o que constitui técnica radical, porém com bons efeitos se aplicada em casos específicos, a exemplo do que ocorre na rotina do tratamento de canal dentário em seres humanos.

$\mathrm{Na}$ medicina eqüina, a neurectomia digital palmar vem sendo utilizada há décadas como forma de aliviar a dor presente em doenças degenerativas podais que não responderam às terapias conservativas (Stashak, 1994). Várias técnicas de neurectomias vêm sendo desenvolvidas, sendo o principal ponto em comum, evidenciado pelos pesquisadores, a preocupação em evitar as complicações póscirúrgicas, que podem ser relativamente freqüentes, como a dessensibilização incompleta da área afetada, a regeneração do segmento de nervo retirado, a formação de neuroma doloroso e, até mesmo, a perda da parede do casco (Hardy, 1992). Dentre elas, a mais tradicional e utilizada no país tem sido a técnica da guilhotina (TG), que consiste na dissecção cuidadosa do nervo, de forma a minimizar o trauma causado nas estruturas circunvizinhas, seguida da secção de um segmento com instrumento cortante extremamente afiado (Turner e McIlwraith, 1985). Essa técnica, apesar de não ser totalmente isenta de complicações, apresentou melhores resultados, quando comparada com técnicas mais atuais, como o uso do laser e a cobertura do coto nervoso com a bainha perineural (Dabareiner et al., 1997).

Outra técnica disponível, porém ainda pouco estudada, foi descrita por Black (1992), que utilizou duas incisões de pele para retirar um fragmento maior de nervo. Segundo esse autor, esse procedimento denominado técnica de stripping (TS), apresentaria a vantagem de uma menor incidência de reinervação e de formação de neuromas dolorosos no pós-operatório. Com a hipótese de que, apesar de demandar maior tempo cirúrgico, a TS resulta em menor ocorrência de complicações no pós-cirúrgico, o objetivo deste trabalho foi estudar animais submetidos a ambas as técnicas, comparando-se as dificuldades transcirúrgicas e as complicações pós-operatórias.

\section{MATERIAL E MÉTODOS}

Quatro éguas sem raça definida, não gestantes, com 8,6 $\pm 1,2$ anos de idade, após tratadas com ivermectina $(0,1 \mathrm{mg} / \mathrm{kg}, \mathrm{PO})$, foram mantidas por um período de adaptação de 15 dias sob dieta balanceada com feno e ração comercial. Os animais apresentavam-se clinicamente hígidos, verificados por meio de exame clínico, hemograma completo e fibrinogênio plasmático. Após 12 horas de jejum sólido, os animais foram pré-medicados com acepromazina $(0,03 \mathrm{mg} / \mathrm{kg}$, IV) e, 30 minutos após, receberam uma solução de éter gliceril guaiacol a 10\% até o decúbito, quando foram intubados segundo técnica descrita por Faleiros et al. (1996). A indução e a manutenção anestésica foram feitas por halotano volatilizado em oxigênio mediante uso de vaporizador compensado, em um circuito circular fechado.

Cada animal foi submetido à cirurgia nos quatro membros em um mesmo tempo cirúrgico. Como padronização, os nervos digitais, lateral e medial, de cada membro, foram submetidos à mesma técnica cirúrgica, sendo que os nervos do membro colateral correspondente, seja torácico ou pélvico, foram submetidos a outra técnica cirúrgica. Entre os grupos, houve igualdade nos números de membros esquerdo e direito, bem como nos pélvico e torácico. Todos os procedimentos cirúrgicos foram realizados pelo mesmo cirurgião.

Nos animais, posicionados em decúbito dorsal, utilizou-se a faixa de Esmarch imediatamente antes de cada procedimento como método de hemostasia preventiva. Na técnica da Guilhotina, para cada ramo, realizou-se uma incisão de pele, de 2 a $3 \mathrm{~cm}$, sobre o plexo digital e em sentido paralelo à borda do tendão flexor profundo, onde se procedeu a dissecação romba do nervo. Após exposto, o nervo foi secionado inicialmente na extremidade distal da incisão. Logo após, uma pinça hemostática foi aplicada no coto proximal, 
que foi esticado e ao mesmo tempo secionado por um bisturi na linha proximal da incisão. $\mathrm{O}$ espaço morto foi reduzido com fio categute 000 e a pele suturada em pontos simples separados com fio de náilon 0 .

$\mathrm{Na}$ técnica de stripping, para cada ramo, foram realizadas duas incisões de pele. Primeiro, realizou-se a incisão distal, de 1,5 a $2 \mathrm{~cm}$, abaxial ao tendão flexor digital profundo e imediatamente proximal à cartilagem colateral, onde foi localizado e exposto o nervo digital. Em seguida, foi realizada a segunda incisão de pele sobre o plexo digital, imediatamente distal à base do sesamóide. O nervo foi novamente dissecado, exposto e posteriormente seccionado com bisturi, quando mantido sob tração, o mais proximal possível, adjacente à base do sesamóide. O coto distal foi então tracionado pela incisão distal até que houvesse sua exposição completa. $\mathrm{O}$ nervo foi seccionado novamente o mais distal possível, próximo da cartilagem colateral. As duas incisões de pele foram suturadas, sem redução do espaço morto, com náilon 0 em pontos simples separados.

Ao final da cirurgia, foram aplicadas bandagens compressivas sobre os membros, sendo substituídas a cada três dias, até a retirada dos pontos que ocorreu com 14 dias. Os animais receberam penicilina sódica $(20.000 \mathrm{UI} / \mathrm{kg}, \mathrm{IM}) \mathrm{e}$ fenilbutazona $(4,4 \mathrm{mg} / \mathrm{kg}$, IV) diariamente, por três dias. Os eqüinos foram mantidos confinados em baia durante as primeiras três semanas, para depois serem soltos em pastagem de gramas nativas, juntamente com outros animais, não receberam, a partir daí, qualquer tipo de cuidado especial. Para se avaliar a facilidade de execução das técnicas, foi mensurada, por meio de cronômetro, a duração de cada procedimento, desde a incisão de pele até o último ponto da sutura. Também foi mensurado, por meio de paquímetro, o comprimento do fragmento de nervo removido.

Para se observar as complicações no pósoperatório imediato, os animais foram acompanhados diariamente nas duas primeiras semanas. Avaliaram-se edema na região da quartela, complicações na cicatrização da ferida e presença de claudicação. Para avaliação em longo prazo foram realizados exames aos dois, três e 14 meses. Além do exame visual para detecção de claudicação, realizou-se um teste de sensibilidade na região dos talões, por meio de estímulo da pele com objeto pontiagudo, mas não perfurante, um teste de sensibilidade no casco, com auxílio de pinça específica, e um teste de presença de neuroma doloroso, pela palpação dos cotos nervosos.

Para as avaliações de edema, presença de claudicação e formação de neuroma doloroso, foram estabelecidos escores de 0 a 4: 0 ausente, 1 discreto, 2 leve, 3 moderado e 4 intenso. Para as avaliações de sensibilidade dos talões e à pinça de casco, os escores foram de 0 a 3: 0 ausente, 1 reação após estímulo intenso, 2 reação normal, 3 reação exacerbada. Essas avaliações foram sempre feitas pelo mesmo pesquisador.

Na comparação entre grupos, os dados paramétricos foram analisados pelo teste $t$ de Student e os não paramétricos pelo teste Mann Whitney. A incidência de sinais de sensibilidade nos talões e no coto nervoso foi analisada, dentro de cada tempo, pelo teste do qui quadrado. Foi considerado o nível de significância de $\mathrm{P}<0,05$ (Sampaio, 2007).

\section{RESULTADOS E DISCUSSÃO}

As cirurgias transcorreram sem complicações, e todos os animais se recuperam normalmente da anestesia. Os tempos médios $( \pm \mathrm{EPM})$ de cirurgia foram de $6,77 \pm 1,21 \mathrm{~min}$ e 7,46 $\pm 1,14 \mathrm{~min}$ para $\mathrm{TG}$ e TS, respectivamente (Fig. 1), e não houve diferença entre eles $(\mathrm{P}=0,11)$. Este achado nega parte da hipótese do presente trabalho e discrepa das observações de Black (1992), de que a TS demanda tempo maior de cirurgia e de anestesia que a TG. Contudo, deve-se observar que todos os procedimentos foram realizados pelo mesmo cirurgião, que, além de treinado, aprimorou suas habilidades em realizar a TS ao longo do período experimental.

Corroborando com os achados do estudo original de Black (1992), o segmento de nervo removido foi quase três vezes maior $(\mathrm{P}<0,001)$ em TS $(8,08 \mathrm{~cm})$, quando comparado com TG $(2,73 \mathrm{~cm})$, conforme representado na Fig. 1. A retirada de um segmento maior tem sido considerada como vantajosa por proporcionar maior período pós-cirúrgico sem retorno da sensibilidade ao membro, devido à ocorrência de reinervação (Black, 1992). 
Tamanho do fragmento

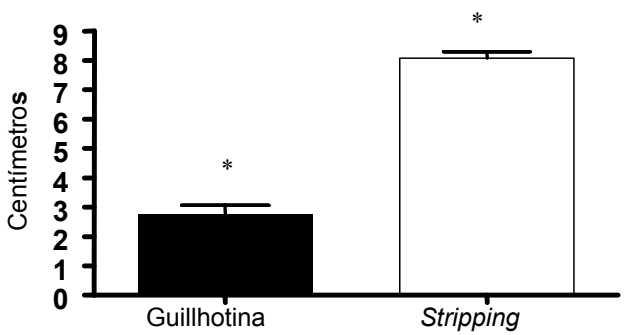

Tempo de cirurgia

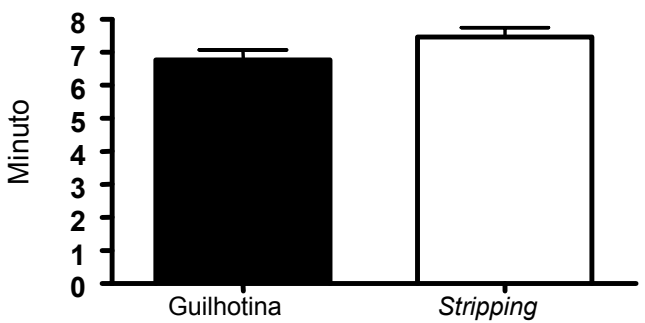

Figura 1. Médias e erros-padrão da média do tamanho do fragmento e do tempo de cirurgia em eqüinos submetidos a duas técnicas de neurectomia digital. *Diferem entre si (teste $\mathrm{t}, \mathrm{P}<0,001$ ).

No pós-cirúrgico imediato, observou-se edema na quartela e no boleto de ausente a discreto nos membros torácicos e de leve a moderado nos membros pélvicos. Edema de quartela e boleto tem sido relatado como uma ocorrência comum nas primeiras duas semanas de pós-cirúrgico (Murray et al., 1994). Apesar da discrepância, não houve diferença $(\mathrm{P}=0,065)$ entre membros pélvicos e torácicos. $\mathrm{O}$ mesmo foi verificado entre as duas técnicas $(\mathrm{P}=0,958)$. Em um dos animais, observou-se secreção purulenta no oitavo dia em ambos os membros pélvicos, cada qual submetido a uma das técnicas. Contudo, a cicatrização da ferida cirúrgica ocorreu de forma satisfatória, tanto nesse animal como nos outros.

Não foi observada claudicação ou sensibilidade anormal no exame de pinça de casco em todos os animais em nenhuma fase do período experimental, com exceção de um animal que teve claudicação discreta e passageira, após ter sido escoiceado aos 30 dias de pós-cirúrgico.
Nessa mesma fase, um dos animais apresentou infecção no sulco central da ranilha, tratada com solução de iodopovidona usada no local e sulfadoxina e trimetropim (15mg/kg, IV). Aos 60 dias, já não existiam mais sinais de infecção.

Aos oito dias de pós-cirúrgico, 68\% (12/16) dos membros operados com a TG e $75 \%$ dos operados com a TS (11/16) ainda apresentavam sensibilidade nos talões. Aos dois meses, apenas um membro em TG e nenhum em TS foram positivos para esse teste (Tab. 1). De forma interessante, o membro que apresentou sensibilidade aos dois meses em TG permaneceu insensível em exames posteriores. Ao final de 14 meses, 37,5\% (6/12) dos membros em TG apresentavam sinais de sensibilidade, três com sensibilidade normal (grau 2) e três com reação após estímulo intenso (grau 1). Em TS, 18,8\% (3/12) dos membros apresentavam sensibilidade normal. O teste de qui quadrado não demonstrou diferença entre tratamentos.

Tabela 1. Número de membros com escores positivos para o teste de sensibilidade nos talões de eqüinos submetidos a duas técnicas de neurectomia digital

\begin{tabular}{|c|c|c|c|c|c|c|c|c|c|c|}
\hline \multirow{2}{*}{$\begin{array}{l}\text { Tempo de } \\
\text { pós } \\
\text { operatório }\end{array}$} & \multicolumn{5}{|c|}{$\begin{array}{l}\text { Escore-guilhotina } \\
\qquad(\mathrm{n}=16)\end{array}$} & \multicolumn{5}{|c|}{$\begin{array}{l}\text { Escore-stripping } \\
\quad(\mathrm{n}=16)\end{array}$} \\
\hline & 1 & 2 & 3 & Total & $\%$ & 1 & 2 & 3 & Total & $\%$ \\
\hline 8 dias & 2 & 9 & 0 & 11 & 68,8 & 3 & 8 & 1 & 12 & 75,0 \\
\hline 2 meses & 0 & 1 & 0 & 1 & 6,3 & 0 & 0 & 0 & 0 & 0,0 \\
\hline 3 meses & 0 & 2 & 0 & 2 & 12,5 & 0 & 0 & 0 & 0 & 0,0 \\
\hline 4 meses & 3 & 0 & 0 & 3 & 18,8 & 1 & 0 & 0 & 1 & 6,3 \\
\hline 14 meses & 3 & 3 & 0 & 6 & 37,5 & 0 & 3 & 0 & 3 & 18,8 \\
\hline
\end{tabular}

Escores para sensibilidade nos talões: $1=$ reação após estímulo intenso; $2=$ reação normal; $3=$ reação exacerbada.

A ausência de sensibilidade cutânea na região dos talões tem sido utilizada como parâmetro para determinar a eficácia da neurectomia digital em eqüinos (Murray et al, 1994). O fato de vários membros ainda apresentarem sensibilidade nesta área, nos primeiros dias após 
a cirurgia, indica que a presença de edema e inflamação, provocados pelo procedimento cirúrgico, promoveu hiperalgesia primária, aumentando a sensibilidade de terminais nervosos circunvizinhos à região desensibilizada (Faleiros et al., 1997). Testes posteriores nos mesmos membros não apresentaram respostas positivas em testes realizados nos meses subseqüentes, o que evidencia que ambas as técnicas foram eficazes.

O aumento de membros com reações positivas ao teste de sensibilidade cutânea nos talões ao final de 14 meses indica ocorrência de reinervação em alguns casos. Segundo Jackman et al. (1993), as possíveis causas de retorno da sensibilidade aos talões são: insensibilização incompleta pela ocorrência de ramos do nervo digital não removidos; presença de um neuroma doloroso, não detectável à palpação; e regeneração nervosa periférica. Uma vez que todos os membros perderam sensibilidade nos primeiros meses após a cirurgia e o retorno de sensibilidade no coto proximal foi apenas discreto, a possibilidade de regeneração nervosa periférica se apresenta como a mais provável. Já foi demonstrado que os nervos periféricos possuem notável capacidade de regeneração em animais de laboratório (Grecco et al., 2003). Em eqüinos, esta capacidade também já foi verificada, contudo existe uma tendência para que ela ocorra de forma desorganizada, com concomitante proliferação de tecido conjuntivo fibroso (Delistoianov et al., 2006).

Apesar de não haver diferença significativa entre os tratamentos ao final, o número de animais com sensibilidade positiva nos talões foi o dobro em TG. Pesquisas futuras, com número maior de animais e com maior tempo de acompanhamento, poderão esclarecer melhor tal diferença. Dabareiner et al. (1997) detectaram retorno da sensibilidade cutânea na face caudal da quartela, em média, 306 dias após a neurectomia pela TG, enquanto Black (1992) observou retorno da sensibilidade em um eqüino após dois anos e meio de neurectomia pela TS.

No teste de sensibilidade do coto nervoso proximal para detecção de formação de neuromas dolorosos (Tab. 2), entre o terceiro e quarto meses do pós-operatório, foram observados $12,5 \%$ (2/16) e $6,3 \%$ (1/16) dos membros com sensibilidade discreta em TG e TS, respectivamente. Contudo, 14 meses após a cirurgia, a porcentagem de membros com sensibilidade aumentou para $56,3 \%$, diferindo estatisticamente da taxa de TS $(\mathrm{P}=0,003)$, que não se alterou.

Tabela 2. Número de membros com escores positivos para o teste de sensibilidade do coto nervoso proximal de eqüinos submetidos a duas técnicas de neurectomia digital

\begin{tabular}{|c|c|c|c|c|c|c|c|c|c|c|c|c|}
\hline \multirow{2}{*}{$\begin{array}{c}\text { Tempo } \\
\text { de pós } \\
\text { operatóri } \\
0\end{array}$} & \multicolumn{6}{|c|}{$\begin{array}{l}\text { Escore-guilhotina } \\
\qquad(n=16)\end{array}$} & \multicolumn{6}{|c|}{$\begin{array}{c}\text { Escore-stripping } \\
(\mathrm{n}=16)\end{array}$} \\
\hline & 1 & 2 & 3 & 4 & $\begin{array}{c}\text { Tota } \\
1\end{array}$ & $\%$ & 1 & 2 & 3 & 4 & Total & $\%$ \\
\hline 2 meses & 2 & 0 & 0 & 0 & 2 & 12,5 & 1 & 0 & 0 & 0 & 1 & 6,3 \\
\hline 3 meses & 2 & 0 & 0 & 0 & 2 & 12,5 & 1 & 0 & 0 & 0 & 1 & 6,3 \\
\hline 4 meses & 9 & 0 & 0 & 0 & 9 & $\begin{array}{l}56,3 \\
*\end{array}$ & 1 & 0 & 0 & 0 & 1 & $6,3^{*}$ \\
\hline 14 meses & 0 & 0 & 0 & 0 & 0 & 0,0 & 0 & 0 & 0 & 0 & 0 & 0,0 \\
\hline
\end{tabular}

Escores para sensibilidade dolorosa no coto nervoso proximal: 0-ausente, 1-discreto, 2-leve, 3-moderado e 4-intenso. *Diferem entre si (teste qui quadrado, $\mathrm{P}=0,003$ ).

A ocorrência de sensibilidade de ausente a discreta em todos os cotos nervosos confirma os achados do exame físico e da avaliação de claudicação, que não detectaram nenhum neuroma doloroso de importância clínica. A ausência clínica de neuroma doloroso também foi observada em animais submetidos à $\mathrm{TG}$ em estudo experimental, após 12 meses da cirurgia
(Dabareiner et al., 1997), e em 10 casos clínicos após 18 meses (Jackman et al., 1993).

\section{CONCLUSÕES}

Ambas as técnicas foram satisfatórias por não apresentarem maiores complicações durante ou após a cirurgia. Contudo, a TG foi tecnicamente 
mais simples de ser executada, pois necessitou apenas de uma incisão e permitiu melhor exposição do campo cirúrgico. A TS apresentou menor potencial para produzir reinervação e neuromas dolorosos por retirar um segmento nervoso até três vezes maior e por apresentar, ao final do experimento, menor número de membros com sensibilidade à palpação do coto proximal.

\section{REFERÊNCIAS BIBLIOGRÁFICAS}

BLACK, J.B. Palmar digital neurectomy: an alternative surgical approach. Proc. Am. Assoc. Equine Pract., v.38, p.429-432, 1992.

DABAREINER, R.M.; WHITE, W.A.; SULLINS, K.E. Comparsion of current techniques for palmar digital neurectomy in horses. Proc. Am. Assoc. Equine Pract., v.43, p.231-232, 1997.

DELISTOIANOV, N.; MACORIS, D.G.; GODOY, R.F. et al. Comparative study of two techniques of digital palmar neurorrhaphy in horses. Arq. Bras. Med. Vet. Zootec., v.58, p.4451, 2006.

FALEIROS, R.R.; ALVES, G.E.S.; MARQUES Jr, A.P. Dor aguda: vias anatômicas, bioquímica e fisiopatologia. Cad. Tec. Esc. Vet. UFMG, n.21, p.5-14, 1997.

FALEIROS, R.R., ALVES, G.E.S., MALM, C. et al. Intubação orotraqueal em eqüinos sob o efeito de acepromazina e éter gliceril guaiacol (resultados parciais). In: CONGRESSO
BRASILEIRO DE MEDICINA VETERINÁRIA, 24, 1996, Goiânia. Anais... Goiânia: SBMV, 1996. p.11.

GRECCO, M.A.S.; LEITE, V.M.; ALBERTONI, W.M. et al. A study on nerve regeneration in tibial nerves of wistars rats, using Fluoro-Gold $\AA$ as a neuronal tracer. Acta Ortop. Bras., v.11, p.225-229, 2003.

JACKMAN, B.R.; BAXTER, G.M.; DORAN, R.E. et al. Palmar digital neurectomy in horses 57 cases (1984-1990). Vet. Surg., v.22, p.285288, 1993.

HARDY, J. Surgical procedures involving the peripheral nerves. In: AUER, J.A. (Ed). Equine surgery. Philadelphia: Saunders, 1992. p.580585.

MURRAY, R.C.; GAUGHAN, E.M.; DeBOWES, R.M. et al. Acute effects of perineural administration of sodium hyaluronate on palmar digital neurectomy sites in horses. Am. J. Vet. Res., v.55, p.1484-1489, 1994.

SAMPAIO, I.B.M. (Ed). Estatística aplicada à experimentação animal. 3.ed. Belo Horizonte: FEP-MVZ, 2007. 264p.

STASHAK, T.S. Claudicação. In: STASHAK, T.S. (Ed). Claudicação em eqüinos segundo Adams. 4.ed. São Paulo: Roca, 1994. p.503-816.

TURNER, A.S.; McILWRAITH, C.W. (Eds). Técnicas cirúrgicas em animais de grande porte. São Paulo: Roca, 1985. 341p. 